\title{
Transmissão oral da doença de Chagas: Uma revisão de literatura
}

\author{
Oral transmission of Chagas disease: A literature review \\ Transmisión oral de la enfermedad de Chagas: Revisión de la literatura
}

Recebido: 03/02/2021 | Revisado: 07/02/2021 | Aceito: 09/02/2021 | Publicado: 17/02/2021

\author{
Luciano Vasconcellos Pacheco \\ ORCID: https://orcid.org/0000-0002-6210-3828 \\ Universidade do Estado da Bahia, Brasil \\ E-mail: lucianofcd@hotmail.com \\ Leonora Souza Santana \\ ORCID: https://orcid.org/0000-0003-3586-9438 \\ Centro Universitário Maurício de Nassau, Brasil \\ E-mail: leonorassantana@gmail.com \\ Breno Cardim Barreto \\ ORCID: https://orcid.org/0000-0002-9897-2443 \\ Instituto Gonçalo Moniz, Brasil \\ Fundação Oswaldo Cruz, Brasil \\ E-mail:brenoc.barreto@hotmail.com \\ Emanuelle de Souza Santos \\ ORCID: https://orcid.org/0000-0002-5213-8197 \\ Instituto de Tecnologia da Saúde, Brasil \\ E-mail: Souza.emanuelle@hotmail.com \\ Cássio Santana Meira \\ ORCID: https://orcid.org/0000-0002-0113-7864 \\ Universidade do Estado da Bahia, Brasil \\ E-mail: cassio.meira@fieb.org.br
}

\begin{abstract}
Resumo
A doença de Chagas, conhecida como tripanossomíase americana, é causada pelo protozoário $T$. cruzi e endêmica da América Latina, no qual representa um dos principais problemas socioeconômicos da região. As vias de transmissão podem ser vetorial, via oral, por transfusão sanguínea, transplante de órgãos, transmissão congênita ou acidentes laboratoriais. A transmissão oral destaca-se por ser uma das principais causas da doença de Chagas Aguda (DCA) em algumas regiões. Dessa maneira, o trabalho visa descrever os principais surtos epidemiológicos de infecção com o $T$. cruzi por contaminação oral com base na literatura e identificar as principais fontes, locais e medidas preventivas tomadas frente a estes surtos causados por contaminação oral. A revisão bibliográfica foi realizada nos bancos de dados: Literatura Latino-Americana e do Caribe em Ciências da Saúde (LILACS), portal Scientific Electronic Library Online (SCIELO) e PubMed. Ao fim da seleção, 13 artigos foram utilizados para o desenvolvimento do estudo. Os trabalhos mostraram que os principais alimentos associados à infecção aguda chagásica foram caldo de cana-deaçúcar, açaí, suco de açaí, água contaminada, suco de palmito e suco de goiaba. As principais localidades acometidas foram o Brasil, Pará, Amapá e Amazonas, e outros países latinos americanos como a Guiana Francesa e Venezuela. Além disso, os estudos apontaram às práticas de controle sanitárias, o processo de cozimento, à adoção de medidas estruturais básicas combinadas com a não utilização de iluminação artificial como possíveis medidas à redução do risco de contaminação nessas áreas. Por fim, esse trabalho traz um levantamento que favorece uma melhor caracterização da importância da transmissão oral da doença de Chagas nos últimos 12 anos na América Latina.
\end{abstract}

Palavras-chave: Doença de chagas; Infecção por Trypanosoma cruzi; Euterpe; Saccharum.

\begin{abstract}
Chagas disease, known as American trypanosomiasis, is caused by the protozoan $T$. cruzi and endemic to Latin America, in which it represents one of the main socioeconomic problems in the region. Transmission routes can be vector, orally, by blood transfusion, organ transplantation, congenital transmission or laboratory accidents. Oral transmission stands out for being one of the main causes of acute Chagas disease (ACD) in some regions. Thus, the work aims to describe the main epidemiological outbreaks of infection with $T$. cruzi by oral contamination based on the literature and to identify the main sources, places and preventive measures taken in the face of these outbreaks caused by oral contamination. The bibliographic review was carried out in the databases: Latin American and Caribbean Literature in Health Sciences (LILACS), Scientific Electronic Library Online portal (SCIELO) and PubMed. At the end of the selection, 13 articles were used for the development of the study. The studies showed that the main foods associated with acute Chagas infection were sugarcane juice, açaí, açaí juice, contaminated water, palm heart juice and guava juice. The main localities affected were Brazil, Pará, Amapá and Amazonas, and other
\end{abstract}


Latin American countries such as French Guiana and Venezuela. In addition, the studies pointed out sanitary control practices, the cooking process, the adoption of basic structural measures combined with not using artificial lighting as possible measures to reduce the risk of contamination in these areas. Finally, this work brings a survey that favors a better characterization of the importance of oral transmission of Chagas disease in the last 12 years in Latin America.

Keywords: Chagas disease; Trypanosoma cruzi Infection; Euterpe; Saccharum.

\section{Resumen}

La enfermedad de Chagas, conocida como tripanosomiasis americana, es causada por el protozoo T. cruzi y es endémica de América Latina, en la que representa uno de los principales problemas socioeconómicos de la región. Las vías de transmisión pueden ser vectoriales, por vía oral, por transfusión de sangre, trasplante de órganos, transmisión congénita o accidentes de laboratorio. La transmisión oral se destaca por ser una de las principales causas de enfermedad de Chagas aguda (ECA) en algunas regiones. Así, el trabajo tiene como objetivo describir los principales brotes epidemiológicos de infección por T. cruzi por contaminación oral con base en la literatura e identificar las principales fuentes, lugares y medidas preventivas tomadas ante estos brotes por contaminación oral. La revisión bibliográfica se realizó en las bases de datos: Literatura Latinoamericana y del Caribe en Ciencias de la Salud (LILACS), Portal en Línea de la Biblioteca Electrónica Científica (SCIELO) y PubMed. Al final de la selección, se utilizaron 13 artículos para el desarrollo del estudio. Los estudios mostraron que los principales alimentos asociados con la infección aguda por Chagas fueron el jugo de caña de azúcar, el açaí, el jugo de açaí, el agua contaminada, el jugo de palmito y el jugo de guayaba. Las principales localidades afectadas fueron Brasil, Pará, Amapá y Amazonas, y otros países latinoamericanos como Guayana Francesa y Venezuela. Además, los estudios señalaron prácticas de control sanitario, el proceso de cocción, la adopción de medidas estructurales básicas combinadas con la no utilización de iluminación artificial como posibles medidas para reducir el riesgo de contaminación en estas áreas. Finalmente, este trabajo trae una encuesta que favorece una mejor caracterización de la importancia de la transmisión oral de la enfermedad de Chagas en los últimos 12 años en América Latina.

Palabras clave: enfermedad de Chagas; Transmisión oral; Infección por Trypanosoma cruzi; Euterpe; Saccharum.

\section{Introdução}

Descrita pelo médico Carlos Chagas em 1909, a doença de Chagas é considerada uma doença negligenciada pela Organização Mundial de Saúde (WHO, 2020). Essa doença é endêmica da América Latina, onde representa um grande problema socioeconômico, visto que afeta principalmente indivíduos que estão em idade economicamente ativa e de regiões pobres (Pérez-Molina; Molina, 2017; Lee et al., 2013; Conteh; Engels; Molyneux, 2010;). A doença, também conhecida como tripanossomíase americana, é causada pelo protozoário hemoflagelado Trypanosoma cruzi, o qual pode ser transmitido vetorialmente através da picada de insetos triatomíneos (Pérez-Molina; Molina, 2017; Chagas, 1922).

Clinicamente, a doença de Chagas pode apresentar duas fases distintas: a fase aguda e a fase crônica. A fase aguda é caracterizada pela presença de tripomastigotas sanguíneos e elevação de anticorpos da classe IgM anti-T.cruzi, podendo os indivíduos nessa fase serem assintomáticos ou apresentarem sintomas de infecção, tais como: febre, anorexia, mal-estar e hepatoesplenomegalia (Benziger; Carmo; Ribeiro, 2017; Lidani et al., 2017; Groom; Protopapas; Zochios, 2017). A fase crônica se caracteriza pela elevação de anticorpos da classe IgG anti-T.cruzi e manifestações clínicas como alterações cardíacas, digestivas ou cardiodigestivas. Além disso, o período compreendido entre as fases aguda e crônica, é denominado de fase indeterminada, na qual encontra-se a maior parte dos indivíduos chagásicos (Simões et al., 2018; Groom; Protopapas; Zochios, 2017; Marin-Neto; Rassi, 2010; Tanowitz et al., 2009).

De acordo com a Organização Mundial de Saúde, estima-se que cerca de 6 a 7 milhões de indivíduos estejam infectados pelo T. cruzi, principalmente na América do Sul (WHO, 2020). No entanto, a busca por melhores condições de vida estimulou o processo migratório de indivíduos infectados, contribuindo para a disseminação da doença para áreas nãoendêmicas (Conners et al., 2016; Coura; Vĩas, 2010). O fato de que a transmissão do T.cruzi pode ocorrer através de diferentes rotas como o consumo de alimentos contaminados, transfusão sanguínea, transplante de órgãos, transmissão congênita ou acidentes laboratoriais contribuem para sua disseminação (Lidani et al., 2017; Coura, 2015; Carlier; Truyens, 2015).

No que se refere à fase aguda, casos de infecção tem sido relatados em regiões não-endêmicas, principalmente em virtude da rota de transmissão oral, devido ao consumo de alimentos contaminados com insetos triatomíneos infectados com o 
T.cruzi (Yoshida, 2008). A contaminação oral é provavelmente a principal rota de transmissão entre animais de ciclo selvagem, visto que muitos deles se alimentam de pequenos insetos como triatomíneos hospedeiros do T.cruzi (Coura, 2015). Já em hospedeiros humanos, esse tipo de transmissão, tem sido reportada desde a década de 1960 (Nóbrega, 2009). No entanto, a contaminação oral só é considerada quando mais de uma pessoa apresenta sintomas agudos de febre, sem causa aparente e que tenham consumido o mesmo tipo de alimento suspeito, como caldo-de-cana e açaí (Santana et al., 2019). Esse cenário tem sido observado principalmente na região amazônica e, menos frequentemente, em outras localidades (Santana et al., 2019; Vargas et al., 2018; Shikanai-Yasuda; Carvalho, 2012).

Diante disso, é necessária uma melhor caracterização do cenário epidemiológico da doença de Chagas, visando reavaliar a disseminação pela contaminação oral em virtude do consumo de alimentos contaminados com o T.cruzi. Nesse contexto, o presente trabalho tem como objetivo revisar a literatura sobre a temática, a fim de descrever os principais surtos epidemiológicos de infecção com o T. cruzi por contaminação oral, bem como identificar as principais fontes, locais e medidas preventivas tomadas frente a estes surtos causados por contaminação oral.

\section{Metodologia}

Para alcançar o objetivo, optou-se pelo método de revisão bibliográfica de natureza sistemática qualitativa. Para tal propósito, foram utilizados os seguintes bancos de dados: Literatura Latino-Americana e do Caribe em Ciências da Saúde (LILACS), portal Scientific Electronic Library Online (SCIELO) e PubMed. A pesquisa das publicações científicas foi realizada nos meses de maio e junho de 2020, por três examinadores.

A revisão foi realizada em cinco etapas, sendo oriunda de uma adaptação do modelo proposto por SOUZA e colaboradores (2010) (Souza; Carvalho; Barrias, 2010). Inicialmente foi delimitada a pergunta norteadora do trabalho: "Qual a contribuição da contaminação oral no atual cenário epidemiológico da doença de Chagas?”. Em seguida foi realizada: a busca na literatura; coleta de dados; análise crítica dos estudos; discussão dos resultados e apresentação da revisão bibliográfica. Em associação, buscou-se seguir os 27 itens recomendados nos Principais Itens para Relatar Revisões Sistemáticas e Meta-Análises (PRISMA) (Moher et al., 2015).

Para efetuar a pesquisa dispondo das plataformas LILACS, SCIELO e Pubmed utilizaram-se os unitermos: " contaminação oral", " alimentos", " transmissão oral", cruzando-os com o unitermo"'Trypanosoma cruzi"'em português, inglês e espanhol, conectadas por " $E$ " "AND" ou ' $Y$ ", de acordo com idioma de pesquisa.

Foram incluídos na revisão os artigos que apresentavam especificidade com o tema, que contivessem os descritores selecionados, e que respeitassem o período supracitado. Os artigos que não indicassem a fonte alimentar da contaminação, revisões bibliográficas de qualquer natureza, trabalhos com uso de modelo animal e aqueles estudos que não foram encontrados na íntegra foram excluídos do estudo.

\section{Resultados e Discussão}

\section{Estudos selecionados}

Dos 384 artigos encontrados, 133 foram excluídos por estarem duplicados. Dos 251 artigos restantes, 157 foram excluídos devido tema incompatível, restando 94 trabalhos. Após leitura, 81 foram excluídos por não identificar fonte alimentar da contaminação, por se tratar de revisão bibliográfica ou utilizar modelo animal experimental. Ao final, 13 artigos foram selecionados e utilizados para construir a amostra do presente estudo. Posteriormente, todos os artigos com critérios de elegibilidade foram lidos na íntegra. Não houve necessidade de submissão ao Comitê de Ética, uma vez que o estudo tem uma abordagem descritiva de revisão de artigos já publicados. O fluxograma de seleção dos artigos para a revisão é descrito na Figura 1. 
Figura 1 - Caracterização dos artigos selecionados para análise. Fonte: Autoria própria. Junho de 2020.
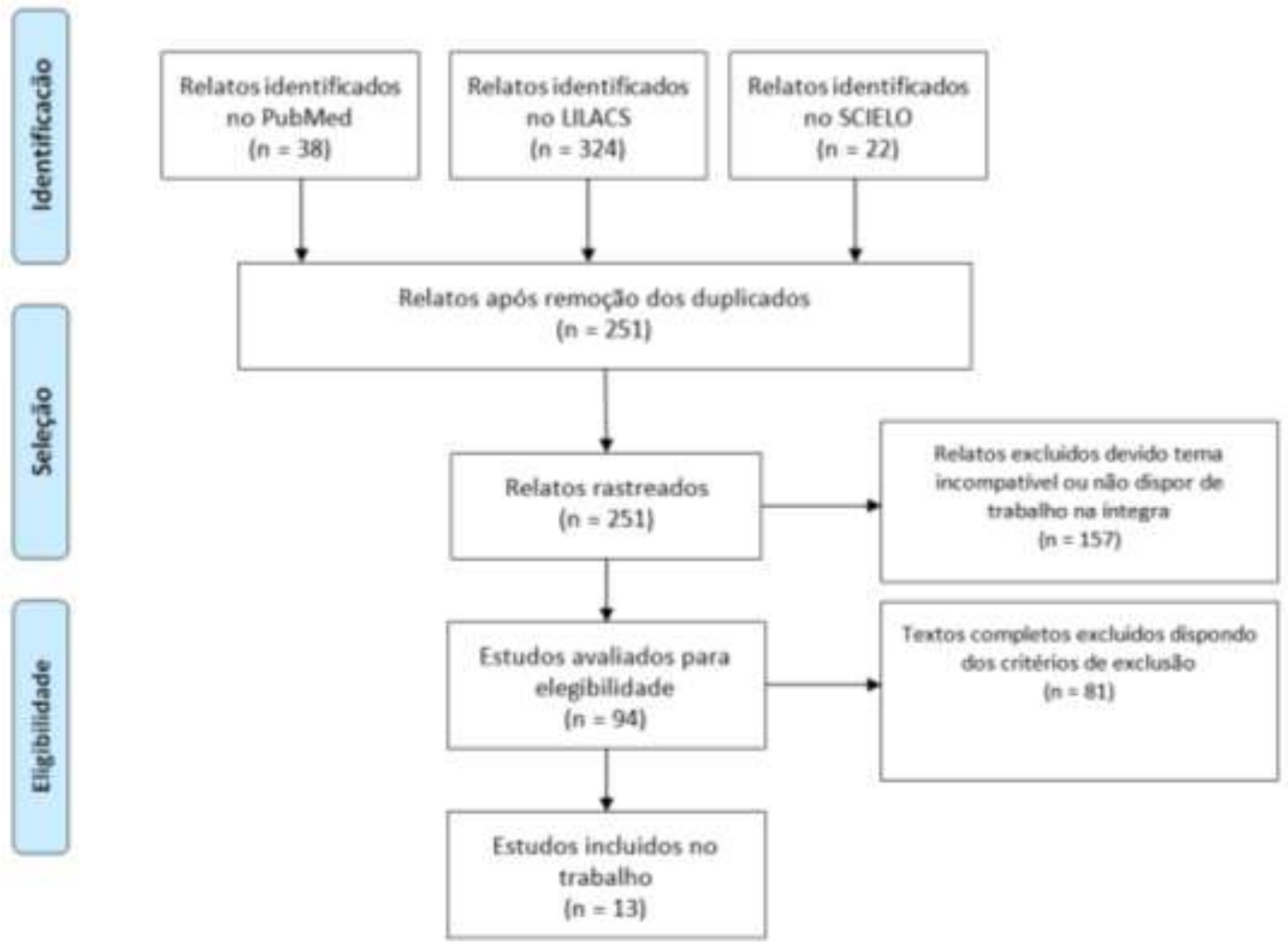

Fonte: Autoria própria.

Alguns trabalhos apresentaram limitações ao associar a contaminação à via oral, de forma que, em um ambiente com presença do vetor e endêmico para doença foi justificado ser um caso agudo por contaminação oral apenas por visualizarem excrementos do triatomíneo associado com a ausência de local de picada recente do inseto, entretanto a amostra não foi testada (Dias et al., 2008). Em contraponto, outros trabalhos avaliaram a presença do parasito em amostras supostamente contaminadas, fortalecendo a hipótese (Santana et al., 2019; Ferreira et al., 2018; Noya et al., 2010).

Por fim, a maioria dos casos associou a infecção com o consumo prévio de algum alimento suspeito por diversos pacientes, os quais apresentaram manifestações clínicas relacionadas com a doença de Chagas aguda (DCA) e ausência de chagoma de inoculação (Vargas et al., 2018; Blanchet et al., 2014; Souza-Lima et al., 2013; Barros et al., 2009; Dias et al., 2008).

Dentre os trabalhos selecionados, nove são estudos epidemiológicos descritivos, do tipo "série de casos" ou "relato de caso". Tal distribuição era esperada, pois ambos os tipos de estudos configuram bons desenhos para a descrição de surtos e epidemias (Parente; Oliveira; De Celeste, 2012). Em adição, foi possível observar que seis dos trabalhos selecionados relataram à ocorrência de óbito, em sua maioria de crianças (Esper et al., 2019; Noya et al., 2017; Noya et al., 2010; Bastos et al., 2010; Dias et al., 2008).

\section{Principais bebidas e alimentos associados à infecção oral por Trypanosoma cruzi}

Nos dias atuais, a transmissão oral apresenta grande importância como uma das principais formas de transmissão da doença de Chagas no Brasil, especialmente na Região Amazônica (Sangenis et al., 2016). A análise dos artigos possibilitou a identificação dos principais alimentos e bebidas associadas à infecção aguda chagásica, sendo eles: caldo de cana-de-açúcar, açaí, suco de açaí, água contaminada, suco de palmito ("vinho" de bacaba-açu) e suco de goiaba. Dentre estes alimentos, o açaí consiste na principal fonte alimentar de contaminação oral, sendo o seu consumo responsável por diversos surto ao longo 
dos últimos anos principalmente na região amazônica e no Pará (Santana et al., 2019; Ferreira et al., 2018; Valente et al., 2009; Nóbrega et al., 2009; Barros et al., 2009). Algumas hipóteses referentes à contaminação por açaí são fundamentadas na atração de triatomíneos contaminados pela luz utilizada durante a extração noturna de polpa de açaí, pela contaminação durante coleta e pela manipulação do açaí sem higiene adequada antes do esmagamento (Santana et al., 2019).

Apesar do consumo de açaí contaminado estar relacionado a diversos surtos de infecção aguda chagásica, o surto descrito que afetou o maior número de pacientes foi associado ao consumo de suco de goiaba contaminado em uma escola de Caracas, Venezuela, no qual 103 crianças foram infectadas, 75\% dos pacientes apresentaram sintomatologia para a doença e uma criança veio a óbito. Este surto afetou um grande número de indivíduos jovens em uma área urbana, e resultou em uma emergência de saúde pública sem precedentes. O diagnóstico e tratamento rápidos evitaram uma maior letalidade (Noya et al., 2010).

Outros dois trabalhos associaram a contaminação oral com o caldo da cana-de-açúcar (Vargas et al., 2018; Bastos et al., 2010). Observou-se que a presença de vetores com positividade para $T$. cruzi próximos ao engenho e associado a fatores de risco, tais como iluminação artificial, podem explicar a contaminação durante a moagem da cana de açúcar. Um fato relevante é que no trabalho anteriormente citado a taxa de triatomíneos infectados com $T$. cruzi foi cerca de dez a quarenta vezes maiores do que é descrito na literatura para outras regiões, tais como Bahia e Ceará (Vargas et al., 2018). Portanto, a alta taxa de triatomíneos infectados configura-se como um agravante e ressalta a necessidade de conhecer a epidemiologia das microrregiões com intuito de propor medidas efetivas de controle.

No período delimitado, não se encontrou casos referentes à contaminação de carne de animais silvestres. Entretanto, o consumo dessa fonte de carne está associado a casos de infecção anteriores ao ano de 2008. Em geral, muitos deles ocorreram em áreas que não se pode descartar a transmissão vetorial e possuem confirmação clínica, e/ou parasitológica, e/ou laboratorial. Dessa maneira, a existência de baixas evidências de transmissão por consumo de carne de animais infectados por T. cruzi configurando-se como um evento raro (Sangenis et al., 2016).

\section{Manifestações clínicas relacionadas à infecção oral por Trypanosoma cruzi}

Sabe-se que a forma tripomastigota do T. cruzi é capaz de invadir e replicar-se na mucosa gástrica, causando uma infecção sistêmica (Bastos et al., 2010). A ingestão de alimentos contaminados pode causar o quadro de DCA, geralmente após cerca de cinco dias da ingestão do alimento, podendo variar de 3 até 22 dias (Esper et al., 2019). Dessa forma, a DCA caracteriza-se por uma síndrome febril aguda associada a sinais e sintomas inespecíficos semelhantes a outras doenças tropicais, como malária, leishmaniose visceral e febre tifóide, o que dificulta a deteç̧ão da mesma (Santana et al., 2019; Esper et al., 2019; Blanchet et al., 2014; Valente et al., 2009).

$\mathrm{Na}$ fase aguda, os parasitas podem ser encontrados no sangue e são evidenciados sintomas tais como: quadro febril, vômito, diarréia, dor no abdômen superior, hepatomegalia, sangramento no trato gastrointestinal superior, edema palpebral bilateral, edema em membros inferiores, taquicardia, dores musculares, alterações no eletrocardiograma e a miocardite grave, esta última associada com pior prognóstico quanto mais novo for o paciente (Esper et al., 2019; Vargas et al., 2018; Blanchet et al.,2014; Souza-Lima et al., 2013; Noya et al., 2010; Barros et al., 2009; Valente et al., 2009; Dias et al., 2008). Duas possibilidades de desfechos clínicos imediatos foram consideradas para os indivíduos em estado grave, sendo eles, a melhora ou óbito nas duas primeiras semanas (Sánchez; Ramírez, 2013). Após a DCA, os pacientes podem progredir para um estado crônico indeterminado e assintomático ou para manifestações clínicas, como doenças cardíacas, sendo a miocardite associada a arritmias e cardiomiopatia dilatada, a evolução mais comum (Toso; Vial; Galanti, 2011). Portanto, devido à inespecificidade dos sintomas para o diagnóstico da DCA, faz-se necessária a utilização de critérios epidemiológicos e testes laboratoriais. Em consonância, Blanchet e colaboradores (2014) relatam que ocorreu atraso no resultado diagnóstico correto devido ao fato de os 
profissionais médicos não estarem familiarizados com a doença de Chagas e os sinais clínicos de febre, astenia, cefaleia e mialgia (inespecíficos) apontarem quase exclusivamente à malária ou dengue, que são endêmicas na região (Blanchet et al., 2014).

\section{Localidades de ocorrência dos surtos causados por ingestão oral de alimentos contaminados por Trypanosoma cruzi}

Em relação às localidades de maior incidência foi possível observar que a grande maioria dos casos de contaminação chagásica aguda oral ocorre no Brasil, em estados do norte do país, tais como Pará, Amapá e Amazonas (Santana et al., 2019; Souza-Lima et al.,2013; Nóbrega et al., 2009; Barros et al., 2009; Valente et al., 2009). Em relação ao Brasil, como podemos observar na Figura 2, os casos de contaminação oral ocorrem na região norte do Brasil, em especial no Pará, cuja maioria dos casos está relacionada ao consumo do açaí (Ferreira et al., 2018; Nóbrega et al., 2009; Barros et al.,2009). Já na Região Nordeste, a Bahia e o Rio Grande do Norte apresentaram casos descritos envolvendo pacientes (Vargas et al., 2018; Bastos et al.,2010; Dias et al., 2008). Por fim, um único trabalho, sem infecções descritas, apenas analisando e identificando amostras de açaí contaminadas com T. cruzi foi realizado no Rio de Janeiro, região sudeste do Brasil (Ferreira et al., 2018).

Figura 1 - Mapa identificando os estados brasileiros que apresentaram casos de contaminação oral registrados no período de 2008 até 2020.

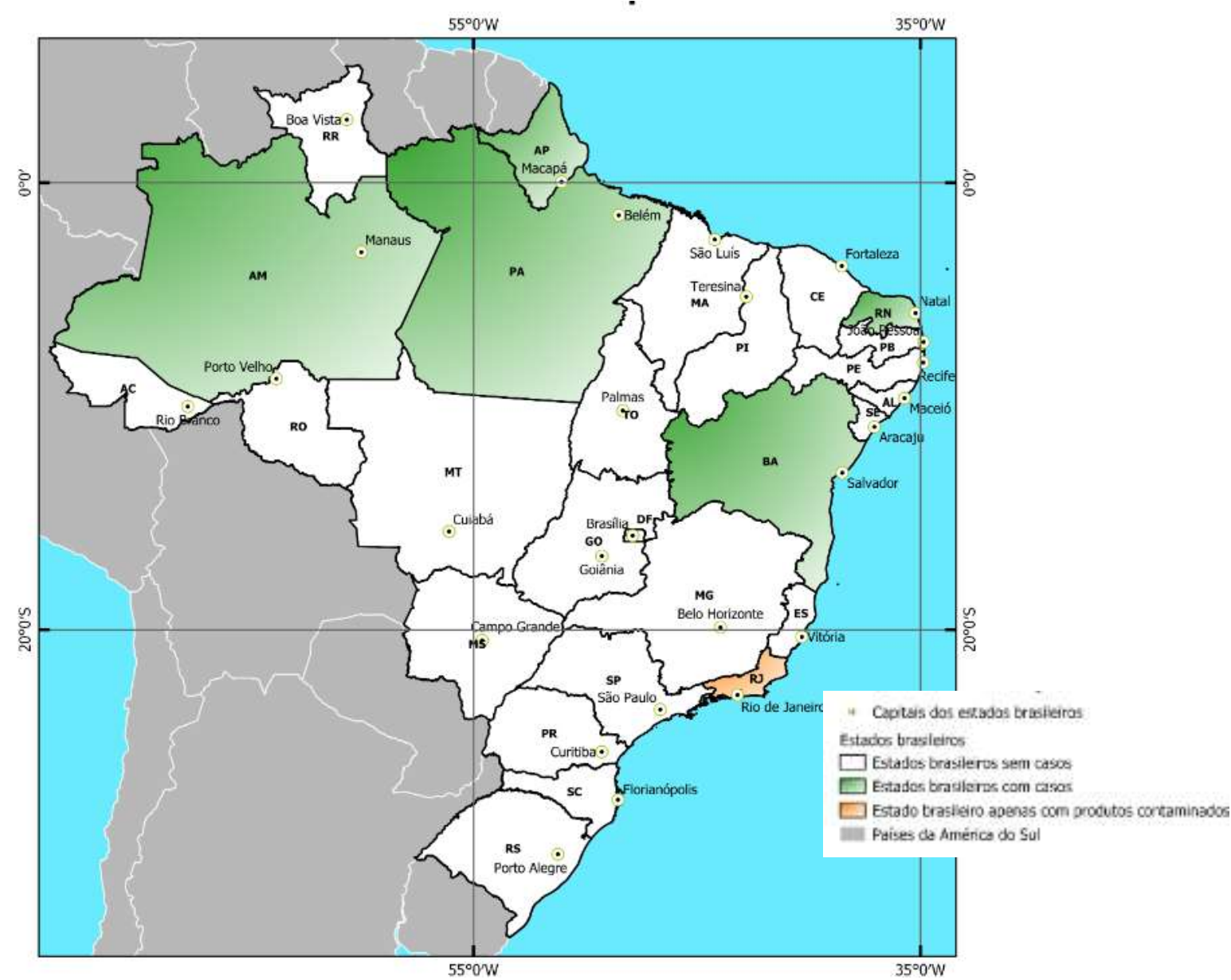

Fonte: Autoria própria.

O mapa foi construído utilizando o software QGIS Desktop, versão 3.14.0. Os estados em verde representam os estados brasileiros com casos confirmados de contaminação chagásica aguda por ingestão de alimentos contendo formas 
parasitárias. O estado em laranja representa os estados brasileiros apenas com produtos contaminados.

\section{Medidas adotadas para combate aos surtos de infecção oral causadas por Trypanosoma cruzi}

Devido ao impacto do número de casos de DCA associados à ingestão de água ou alimentos contaminados, medidas profiláticas para evitar a contaminação oral necessitam ser formuladas e implantadas. A utilização de substâncias com atividade tripanocida, como solução de hipoclorito a 1\%, álcool 70\% e a violeta de genciana apresentam eficácia descrita para eliminação de T.cruzi (Sánchez; Ramírez, 2013). É importante ressaltar, que a violeta de genciana também é usada em bancos de sangues, para evitar a transmissão via transfusão sanguínea (Dias et al., 2008). Contudo, estas práticas de controle sanitárias ainda não são utilizadas pelos produtores de médio e pequeno porte, que acabam sendo os principais responsáveis pelos casos de contaminação oral na região Amazônica (Ferreira et al., 2018).

Referente às carnes, estudos de características biológicas e fisico-químicas demonstraram que o T. cruzi é eliminado após o processo de cocção com temperatura acima de $45^{\circ} \mathrm{C}$, constituindo-se em uma medida profilática eficaz (Sangenis et al., 2016). Portanto, a educação sobre a contaminação de alimentos crus, deve ser fornecida à população para evitar surtos de DCA (Valente et al., 2009). A contaminação experimental de alimentos com T. cruzi aponta que a sobrevivência do parasita varia de acordo com o tipo de alimento e a presença ou ausência de refrigeração, de maneira que longa sobrevivência do parasita pode estar associada ao congelamento do alimento na presença de crioprotetores, provavelmente presentes no teor de gordura (Santana et al., 2019; Ferreira et al., 2018). Dessa forma, a efetividade do congelamento diverge entre os autores. Alguns trabalhos apontam manutenção da virulência após $26 \mathrm{~h}$ de congelamento e outros afirmam que o T. cruzi é inativado após congelamento por $2 \mathrm{~h} \mathrm{a}-20{ }^{\circ} \mathrm{C}$ (Ferreira et al., 2018). Por fim, outro trabalho define que a ingestão do açaí congelado é protetora quando consumidos produtos derivados dessa fruta (Nóbrega et al., 2009).

A falta de uniformidade na produção de polpa e produtos à base de açaí combinados com a inadequada qualidade sanitária, configurou o açaí como o principal alimento contaminado. Nesse sentido, a pasteurização, técnica criada por Louis Pasteur que consiste na esterilização do alimento através do aquecimento do mesmo e rápido resfriamento posterior, vem sendo utilizada com sucesso visando à eliminação de células parasitadas, em especial para a comercialização do açaí (Ferreira et al., 2018; Dias et al., 2008). Porém o desenvolvimento de ferramentas analíticas para o controle de qualidade desses produtos ainda é um desafio (Ferreira et al., 2018).

Acerca do consumo de água, apesar de não ser uma das principais formas de infecção, foi observado que algumas famílias não possuíam água encanada e a estocava em reservatório sem cobertura adequada, de maneira que, foi encontrada contaminação por excremento de triatomíneos infectados (Dias et al., 2008). Associado a medidas de saneamento básico e controle do vetor, que deveriam ser realizadas pelo poder público, a cobertura dos recipientes de água poderia ser uma medida simples adotada, dentro da realidade da família apresentada.

Além disso, a ausência de proteção contra roedores e insetos, bem como a realização das atividades com iluminação elétrica no período da madrugada foi observado em um dos estudos Dessa forma, a falta de estrutura, associada à grande quantidade de triatomíneos capturados na região, pode ter sido um dos fatores facilitadores da contaminação da cana-de-açúcar utilizada (Vargas et al., 2018). Dessa forma, as medidas estruturais básicas combinadas com a não utilização de iluminação artificial durante o processamento da cana-de-açúcar apresentam-se como ações simples e que podem ser executadas para reduzir o risco de contaminação, levando-se em consideração que a iluminação artificial atrai diversos insetos, inclusive os triatomíneos. Todos os artigos inseridos no presente estudo e alguns dos achados mais importantes estão descritos no Quadro 1. 
Quadro 1 - Caracterização dos artigos selecionados para análise.

\begin{tabular}{|c|c|c|c|c|c|}
\hline Autor & Relação com o objetivo & Tipo de estudo & $\begin{array}{c}\text { Fonte associado } \\
\text { à infecção }\end{array}$ & $\begin{array}{l}\text { Número de } \\
\text { casos }\end{array}$ & Relato de óbito \\
\hline Dias et al., 2008 & $\begin{array}{c}\text { Relato de indivíduos } \\
\text { contaminados por T. cruzi no } \\
\text { Sudoeste da Bahia devido à } \\
\text { ingestão de água contaminada } \\
\text { com fezes de triatomíneos } \\
\text { infectados. }\end{array}$ & Série de casos & $\begin{array}{c}\text { Água } \\
\text { contaminada }\end{array}$ & 7 & $\operatorname{Sim}(2)$ \\
\hline Valente et al., 2009 & $\begin{array}{l}\text { Relato de surto de contaminação } \\
\text { oral no Mazagão, Amapá em } \\
1996.17 \text { casos de infecção foram } \\
\text { confirmados e uma grande } \\
\text { variedade de cepas de } T \text {. cruzi foi } \\
\text { confirmada circulando na região. }\end{array}$ & Série de casos & Suco de açaí & 17 & Não \\
\hline Nóbrega et al., 2009 & $\begin{array}{l}\text { Investigação de casos de } \\
\text { contaminação oral por } T \text {. cruzi } \\
\text { associados ao consumo de açaí no } \\
\text { estado do Pará (Barcarena). O } \\
\text { intuito do artigo foi tentar } \\
\text { explanar o motivo de um surto } \\
\text { prévio de doença de Chagas } \\
\text { aguda em } 178 \text { pacientes. }\end{array}$ & $\begin{array}{c}\text { Caso-controle e } \\
\text { Coorte }\end{array}$ & Açaí & 11 & Não \\
\hline Barros et al.,2009 & $\begin{array}{c}\text { Artigo relatando } 25 \text { casos de } \\
\text { infecção por } T \text {. cruzi, nos } \\
\text { municípiosde Breves e Bagre no } \\
\text { estado do Pará. }\end{array}$ & Coorte & $\begin{array}{l}\text { Suco ou pasta de } \\
\text { açaí (sem } \\
\text { confirmação } \\
\text { específica) }\end{array}$ & 25 & Não \\
\hline Noya et al., 2010 & $\begin{array}{c}\text { Artigo descrevendo o maior surto } \\
\text { de doença de Chagas causado por } \\
\text { contaminação oral (Caracas- } \\
\text { Venezuela). }\end{array}$ & $\begin{array}{c}\text { Caso-controle e } \\
\text { Coorte }\end{array}$ & Suco de goiaba & 103 & $\operatorname{Sim}(1)$ \\
\hline Bastos et al., 2010 & $\begin{array}{c}\text { Artigo relatando } 13 \text { casos de } \\
\text { contaminação oral nas cidades de } \\
\text { Macaúbas e Ibipitanga (Estado } \\
\text { Bahia). }\end{array}$ & Série de casos & $\begin{array}{l}\text { Água } \\
\text { contaminada e } \\
\text { caldo de cana- } \\
\text { de-açucar }\end{array}$ & 13 & $\operatorname{Sim}(2)$ \\
\hline $\begin{array}{l}\text { Souza-Lima et al., } \\
2013\end{array}$ & $\begin{array}{l}\text { Artigo relatando casos de infecção } \\
\text { na Região do Rio Negro } \\
\text { Amazônia. Todos os pacientes } \\
\text { consumiram açai da zona rural de } \\
\text { Monte Alegre (Pará). }\end{array}$ & Série de casos & Suco de açaí & 14 & Não \\
\hline Blanchet et al., 2014 & $\begin{array}{c}\text { Primeiro relato de infecção aguda } \\
\text { por ingestão de suco de palmito } \\
\text { Oenocarpusbacapa, registrando } \\
\text { na Guiana Francesa. }\end{array}$ & Série de casos & $\begin{array}{l}\text { Suco de palmito } \\
\text { "bacaba-açu", }\end{array}$ & 8 & Não \\
\hline
\end{tabular}




\begin{tabular}{|c|c|c|c|c|c|}
\hline Noya et al.,2017 & $\begin{array}{l}\text { Artigo relata o décimo primeiro } \\
\text { surto de contaminação oral na } \\
\text { Venezuela e demonstra a via de } \\
\text { transmissão vertical em gestantes } \\
\text { infectadas por ingestão de } \\
\text { alimentos contaminados. }\end{array}$ & Relato de casos & $\begin{array}{c}\text { Mandioca e chá } \\
\text { gelado caseiro }\end{array}$ & 3 & $\operatorname{Sim}(1)$ \\
\hline Ferreira et al., 2018 & $\begin{array}{l}\text { Os autores investigaram diversas } \\
\text { amostras de açaí comercializados } \\
\text { no Pará e no Rio de Janeiro e } \\
\text { encontram DNA do } T \text {. cruzi em } \\
14 \text { amostras, mostrando que a } \\
\text { ingestão deste alimento pode de } \\
\text { fato ocasionar surtos de } \\
\text { contaminação oral. }\end{array}$ & $\begin{array}{c}\text { Corte } \\
\text { transversal } \\
\text { descritivo }\end{array}$ & Açaí & 0 & Não \\
\hline Vargas et al., 2018 & $\begin{array}{l}\text { O artigo relata um surto de } \\
\text { contaminação oral aguda na } \\
\text { mesorregião Oeste Potiguar, Rio } \\
\text { Grande do Norte, relacionado a } \\
\text { ingestão de caldo de cana-de- } \\
\text { açúcar. }\end{array}$ & Serie de casos & $\begin{array}{l}\text { Caldo de cana- } \\
\text { de-açucar }\end{array}$ & 18 & $\operatorname{Sim}(3)$ \\
\hline Santana et al., 2019 & $\begin{array}{l}\text { O artigo relata um surto da doença } \\
\text { de Chagas aguda, envolvendo } 10 \\
\text { pacientes na Amazônia brasileira } \\
\text { (municípios de Manaus e Lábrea), } \\
\text { sendo relatado o suco de açaí } \\
\text { como fonte de infecção. Foram } \\
\text { encontradas tripomastigotas em } \\
\text { amostras de suco. }\end{array}$ & Série de casos & Açaí & 10 & Não \\
\hline Esper et al., 2019 & $\begin{array}{l}\text { O artigo reporta um caso de } \\
\text { infecção aguda de doença de } \\
\text { chagas, envolvendo uma criança } \\
\text { de } 22 \text { meses, causada pelo } \\
\text { consumo de suco de bacaba-açu } \\
\text { na região norte do Brasil (Pará). }\end{array}$ & Relato de Caso & $\begin{array}{l}\text { Suco de palmito } \\
\text { "bacaba-açu", }\end{array}$ & 1 & $\operatorname{Sim}(1)$ \\
\hline
\end{tabular}

Fonte: Autoria própria.

\section{Considerações Finais}

Com o presente estudo, foi possível realizar uma melhor caracterização da importância da transmissão oral da doença de Chagas nos últimos 12 anos na América Latina. Os casos relatados estão distribuídos em três países latinos americanos, sendo o Brasil o epicentro de casos. Os principais alimentos e bebidas associadas à infecção chagásica são: caldo de cana-deaçúcar, açaí, suco de açaí, carne, água contaminada, suco de palma e suco de goiaba. A identificação das localidades e fontes alimentares é vital para o desenvolvimento de um controle sanitário adequado para evitar surtos da doença de Chagas causados pela ingestão de alimentos ou bebidas contaminadas como formas parasitárias. Ademais, a notificação e compilação dos casos de transmissão da doença de Chagas por via oral, são imprescindíveis para monitorização do impacto futuro dessa problemática e execução de novos trabalhos acerca do tema. 


\section{Referências}

Barros, M. B. H., de Paula, C. M., Freitas, D. R. C., Das Neves P. A. Y., da Costa, V. V., Valente, S. A., Costa, E. G., \& Sobel, J. (2009). Investigation of two outbreaks of suspected oral transmission of acute Chagas disease in the Amazon region, Pará State, Brazil, in 2007. Trop Doct. 39(4):231-2.

Bastos, C. J. C., Aras, R., Mota, G., Reis, F., Dias, J. P., de Jesus R. S., Freire, M. S., Araújo, E. G., Prazeres, J., \& Grassi M. F. R. (2010). Clinical outcomes of thirteen patients with acute chagas disease acquired through oral transmission from two urban outbreaks in Northeastern Brazil. PLoS Negl Trop Dis. $4(6): 16-7$.

Benziger, C. P., Carmo, G. A. L., \& Ribeiro, A. L. P. (2017). Chagas Cardiomyopathy: Clinical Presentation and Management in the Americas. Cardiol Clin. 35(1):31-47.

Blanchet, D., Brenière, S. F., Schijman, A. G., Bisio, M., Simon, S., Véron, V., Mayenced, C., Demar-Pierrea, M., Djossoue, F., \& Aznar, C. (2014). First report of a family outbreak of Chagas disease in French Guiana and posttreatment follow-up. Infect Genet Evol. 28:245-50.

Carlier, Y., \& Truyens, C. (2015) Congenital Chagas disease as an ecological model of interactions between Trypanosoma cruzi parasites, preg nant women, placenta and fetuses. Acta Trop. 103-115.

Chagas, C. (1922). Descoberta do Trypanosoma cruzi e verificação da tripanossomíase americana: retrospecto histórico. Memórias do Instituto Oswaldo Cruz. Vol. 15, p. 67-76.

Conners, E. E., Vinetz, J. M., Weeks, J. R., \& Brouwer, K. C. (2016). A global systematic review of Chagas disease prevalence among migrants Graphical abstract HHS Public Access. Acta Trop. 156 (Mc 0507):68-78.

Conteh, L., Engels, T., \& Molyneux, D. H. (2010). Socioeconomic aspects of neglected tropical diseases. Lancet. 375(9710):239-47.

Coura, J. R., \& Vĩas, P. A. (2010). Chagas disease: A new worldwide challenge. Nature. Jun 24,465(7301):S6-7.

Coura, J. R. (2015). The main sceneries of chagas disease transmission. The vectors, blood and oral transmissions - A comprehensive review. Mem Inst Oswaldo Cruz. 110(3):277-82.

Dias, J. P., Bastos, C., Araújo, E., Mascarenhas, A. V., Netto, E. M., Grassi, F., Silva, M., Tatto, E., Mendonça, J., Araújo, R. F., Shikanai-Yasuda, M. A., \& Aras, R. (2008). Acute Chagas disease outbreak associated with oral transmission. Rev Soc Bras Med Trop. 41(3):296-300.

Esper, H. R., de Freitas, V. L. T., Assy, J. G. P. L., Shimoda, E. Y., Berreta, O. C. P., Lopes, M. H., \& França F. O. S. (2019). Fatal evolution of acute chagas disease in a child from northern Brazil: Factors that determine poor prognosis. Rev Inst Med Trop Sao Paulo.61:5-9.

Ferreira, R. T. B., Cabral, M. L., Martins, R. S., Araujo, P. F., Silva, S. A., Britto, C. Branquinho, M. R., Cardarelli-Leite, P., \& Moreira, O. C. (2018). Detection and genotyping of Trypanosoma cruzi from açai products commercialized in Rio de Janeiro and Pará, Brazil. Parasites and Vectors. 11(1):1-11.

Groom, Z. C., Protopapas, A. D., \& Zochios, V. (2017). Tropical diseases of the myocardium: A review. Int J Gen Med.10:101-11.

Lee, B. Y., Bacon, K. M., Bottazzi, M. E., \& Hotez, P. J. (2013). Global economic burden of Chagas disease: A computational simulation model. Lancet Infect Dis.13(4):342-8.

Lidani, K. C. F., Bavia, L., Ambrosio, A. R., \& de Messias-Reason, I. J. (2017). The complement system: A prey of Trypanosoma cruzi. Front Microbiol. $8(\mathrm{APR}): 1-14$.

Marin-Neto, J. A., \& Rassi, A. (2010). Update on Chagas Heart Disease on the First Centennial of Its Discovery. Rev Española Cardiol (English Ed. 62(11):1211-6.

Moher, D., Shamseer, L., Clarke, M., Ghersi, D., Liberati, A., Petticrew, M., \& Shekelle, P. (2015). Preferred reporting items for systematic review and metaanalysis protocols (PRISMA-P) 2015 statement. Syst Rev. January:1-9.

Nóbrega, A. A., Garcia, M. H., Tatto, E., Obara, M. T., Costa, E., Sobel, J., \& Araujo W. N. (2009). Oral transmission of chagas disease by consumption of Açaí palm fruit, Brazil. Emerg Infect Dis. Apr, 15(4): 653-655.

Noya, B. A., Díaz-Bello, Z., Colmenares, C., Ruiz-Guevara, R., Mauriello, L., Zavala-Jaspe, R., Suarez, J. A., Abate, T., Naranjo, L., Paiva, M., Rivas, L., Castro, J., Márques, J., Mendoza, I., Acquatella, H., Torres, J., \& Noya, O. (2010). Large Urban Outbreak of Orally Acquired Acute Chagas Disease at a School in Caracas, Venezuela. J Infect Dis. 201(9):1308-15.

Noya, B. A., Pérez-Chacón G, Díaz-Bello, Z., Dickson, S., Muñoz-Calderón, A., Hernández, C., Pérez, Y., Mauriello, L., \& Moronta, E. (2017). Description of an oral chagas disease outbreak in Venezuela, including a vertically transmitted case. Mem Inst Oswaldo Cruz. 112(8):569-71.

Parente, R. C. M., Oliveira, M. A. P., \& De Celeste, R. K. (2012). Relatos e Série de Casos na Era da Medicina Baseada em Evidência. Brazilian J os Videoendoscopic Surg.3:67-70.

Sangenis, L. H. C., Nielebock, M. A. P., da Silva, S. C., da Silva M. C. C., \& Bento, G. M. R. (2016). Transmissão da doença de Chagas por consumo de carne de caça: Revisão sistemática. Rev Bras Epidemiol.19(4):803-11.

Pérez-Molina, J. A., \& Molina, I. (2017). Chagas disease. Lancet. 391(10115):82-94.

Sánchez, L. V., \& Ramírez, J. D. (2013). Congenital and oral transmission of American trypanosomiasis: An overview of physiopathogenic aspects. Parasitology. 140(2):147-59.

Toso, M. A., Vial, U. F., \& Galanti, N. (2011). Transmisión de la enfermedad de Chagas por vía oral. Rev Med Chil. 139: 258-266 
Santana, R. A. G., Guerra, M. G. V. B., Sousa, D. R., Couceiro, K., Ortiz, J. V., Oliveira, M., Ferreira, L.S., Souza, K. R., Tavares, I. C., Morais, R. F., Silva, G. A. V., Melo, G. C., Vergel, G. M., Albuquerque, B. C., Arcanjo, A. R. L., Monteiro, W. M., Ferreira, J. B. B., Lacerda, M. V. G., Silveira, H., \& Guerra, J. A. O. (2019). Oral transmission of Trypanosoma cruzi, Brazilian Amazon. Emerg Infect Dis. 25(1): 132-135.

Shikanai-Yasuda, M. A., \& Carvalho, N. B. (2012). Oral transmission of chagas disease. Clin Infect Dis. Mar,54(6):845-52. 10.1093/cid/cir956.

Simões, M. V., Romano, M. M. D., Schmidt, A., Martins, K. S. M., \& Marin-Neto, J. A. (2018). Cardiomiopatia da Doença de Chagas. Int J Cardiovasc Sci $.31(2): 173-89$.

Souza, W., Carvalho, T. M. U., \& Barrias, E. S. (2010). Review on Trypanosoma cruzi : Host Cell Interaction. Int J Cell Biol. 2010:1-18.

Souza-Lima, R. C., Barbosa, M. G. V., Coura, J. R., Arcanjo, A. R. L., Nascimento, A. S., Ferreira, J. M. B. B., Magalhães, L. K., Albuquerque, B. C., Araújo, G. A. N., \& Guerra, J. A. O. (2013). Outbreak of acute Chagas disease associated with oral transmission in the Rio Negro region, Brazilian Amazon. Rev Soc Bras Med Trop.46(4):510-4.

Tanowitz, H. B., Machado, F. S., Jelicks, L. A., Shirani, J., Carvalho, A. C., Spray, D. C., Factor, S. M., Kirchhoff, V. L., \& Weiss, L. M. (2009). Perspectives on Trypanosoma cruzi-Induced Heart Disease (Chagas Disease). Prog Cardiovasc Dis. 51(6):524-39.

Valente, S. A. S., da Costa, V. V., das Neves, P. A. Y., de Jesus, B. C. M., dos Santos, M. P., Miranda, C. O. S., Cuervo, P., \& Fernandes, O. (2009). Analysis of an acute Chagas disease outbreak in the Brazilian Amazon: human cases, triatomines, reservoir mammals and parasites. Trans $R$ Soc Trop Med Hyg. 103(3):291-7.

Vargas, A., Malta, J. M. A. S., da Costa, V. M., Cláudio, L. D. G., Alves, R. V., Cordeiro, G. S., Aguir L. M. A., \& Percio, J. (2018). Investigação de surto de doença de Chagas aguda na região extra-amazônica, Rio Grande do Norte, Brasil, 2016. Cad Saude Publica. 34(1):e00006517.

World Health Organisation. (2020). Chagas disease (also known as American trypanosomiasis). Chagas Dis Fact Sheets [Internet]. https://www.who.int/en/news-room/fact-sheets/detail/chagas-disease-(american-trypanosomiasis)

Yoshida, N. (2008). Trypanosoma cruzi infection by oral route. How the interplay between parasite and host components modulates infectivity. Parasitology International Jun,57(2):105-9. 